\title{
EL MÉTODO SINGAPUR PARA EL APRENDIZAJE DE LAS MATEMÁTICAS. ENFOQUE Y CONCRECIÓN DE UN ESTILO DE APRENDIZAJE
}

\author{
Alberto Zapatera Llinares \\ Universidad Cardenal Herrera CEU. \\ Departamento de Ciencias de la Educación \\ Alberto.zapatera@uchceu.es
}

Recepción Artículo: 9 julio 2020

Admisión Evaluación: 12 octubre 2020

Informe Evaluador 1: 25 noviembre 2020

Informe Evaluador 2: 23 noviembre 2020

Aprobación Publicación: 27 noviembre 2020

\section{RESUMEN}

Los excelentes resultados obtenidos por Singapur en las últimas pruebas PISA 2015 y TIMMS 2015, han provocado un gran interés por el denominado Método Singapur de aprendizaje de las matemáticas. El marco curricular de Singapur tiene como centro de aprendizaje la resolución de problemas matemáticos y se basa en cuatro aspectos fundamentales: el enfoque CPA (concreto - pictórico - abstracto), el currículo en espiral, las variaciones sistemática y perceptual y la comprensión relacional. Estos aspectos se fundamentan en las ideas de Jerome Bruner, Zoltan Dienes y Richard Skemp. En este trabajo, tras presentar el sistema educativo de Singapur y el marco curricular, se estudian las aportaciones de estos autores y sus concreciones en el Método Singapur y se extraen una serie de conclusiones para su adaptación.

Palabras clave: aprendizaje; programa de estudios; método de enseñanza; comprensión; desarrollo cognitivo

\footnotetext{
ABSTRACT

The Singapore Method for the mathematics learning. Approach and concretion of a learning style. The latest mathematics assessment tests, PISA 2015 and TIMMS 2015 have caused a great interest in the Singapore Method of Mathematics Learning. The Singapore curriculum framework, which has as a learning center the resolution of mathematical problems and which is developed through four fundamental aspects: the CPA approach (Concrete - Pictorial - Abstract), the spiral curriculum, systematic and perceptual variations and relational understanding. The Singapore Method is based on the ideas of Jerome Bruner, Zoltan Dienes and Richard Skemp. In this work, after presenting the Singapore educational system and the curricular framework, the contributions of these authors and their concretions in the Singapore Method of learning mathematics are studied.

Keywords: learning; curriculum; teaching method; comprehension; cognitive development
} 


\section{EL MÉTODO SINGAPUR PARA EL APRENDIZAJE DE LAS MATEMÁTICAS. ENFOQUE Y CONCRECIÓN DE UN ESTILO DE APRENDIZAJE}

\section{INTRODUCCIÓN}

Aunque los resultados en el área de matemáticas obtenidos por estudiantes portugueses y españoles en las evaluaciones internacionales se aproximan a la media de los países de la OCDE, muestran evidencias de un alto margen de crecimiento, especialmente si los comparamos con los obtenidos por los estudiantes de Singapur.

En el último Informe PISA (Programa Internacional para la Evaluación de Estudiantes) del año 2015, que estudia el rendimiento de estudiantes de 15 años, Singapur obtuvo 564 puntos con un $60 \%$ de los alumnos en Ios niveles superiores, mientras que Portugal y España obtuvieron respectivamente 492 y 486 puntos, con solo un $30 \%$ y un $25 \%$ de alumnos en los niveles superiores. Esta diferencia supone un retraso de los estudiantes portugueses y españoles de casi dos cursos respecto a los de Singapur (Figura 1).

Figura 1. Comparación Singapur y España en PISA 2015 y TIMSS 2015

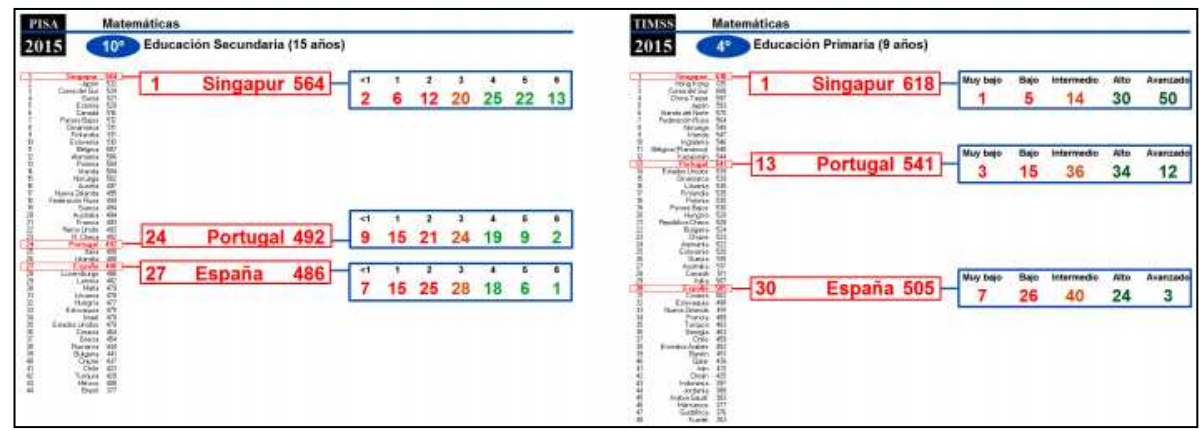

El estudio TIMSS (Estudio Internacional de Tendencias en Matemáticas y Ciencias) del mismo año, que analiza el rendimiento de estudiantes de $4^{0}$ de Educación Primaria, confirman esta tendencia (Figura 1).

El análisis de estos resultados evidencia, por una parte, el excelente rendimiento que le ha proporcionado a Singapur el cambio realizado en la enseñanza de las matemáticas y, por otra parte, la necesidad de países como España y Portugal de realizar una revisión profunda de sus programas de estudios para mejorar el rendimiento de sus estudiantes (Alves, 2018; Mosquera y Salazar, 2014; Santaolalla, 2009). Una opción podría ser adaptar, al menos parcialmente, la metodología utilizada por Singapur, como están haciendo 42 países, entre ellos Estados Unidos, Holanda, Brunei, Australia, India, Tailandia, Libia o Chile.

\section{EL SISTEMA EDUCATIVO Y MARCO CURRICULAR DE SINGAPUR}

Singapur es una república del sudeste asiático con más de cinco millones de habitantes que, tras independizarse de Malasia en 1965, ha pasado de ser un país pobre y sin recursos naturales a convertirse en una economía industrial moderna. Uno de los impulsores de este crecimiento ha sido su sistema educativo que lleva dos décadas desarrollando el programa "Escuelas que piensan, nación que aprende" y que le ha promocionado a los primeros puestos en las últimas evaluaciones internacionales de rendimiento escolar. Este interés de Singapur por la educación se concreta en su gasto en educación que supone el 20\% del presupuesto total del país, mientras que en Portugal o España no alcanza el 10\%.

El sistema educativo de Singapur está orientado a desarrollar aptitudes, valores y habilidades en los estudiantes que les permitan enfrentarse a los cambios de la sociedad y al desarrollo de tecnologías. Para ello propone metodologías que priman el aprendizaje sobre la memoria y que procuran que el estudiante construya su propio aprendizaje explorando y buscando distintas estrategias.

Aunque las reformas de los últimos años buscan ofrecer respuestas individuales a cada estudiante adaptando el itinerario académico a sus capacidades, el sistema educativo está basado en la meritocracia y la competitividad: 
antepone la eficiencia sobre la equidad, clasifica a los estudiantes según su rendimiento académico y promociona a los que obtienen mejores resultados.

El sistema educativo en Singapur se estructura en cuatro niveles básicos: Preescolar, Primaria, Secundaria y Post-secundaria. (Figura 2). Los niños comienzan su escolarización en Preescolar a los cuatro años y a los seis años pasan a la Educación Primaria que tiene una duración de seis años. Al final de la Educación Primaria, los estudiantes se someten a un examen que determina, según sus capacidades, los estudios que seguirán después en la Educación Secundaria: Especial destinada a estudiantes altamente dotados, Exprés destinada también a estudiantes con un alto nivel e impartida en su lengua materna o Normal destinada a la mayoría de estudiantes. Al final de la Secundaria, Ios estudiantes realizan otro examen y, según el resultado, son canalizados con mayor precisión hacia las distintas modalidades de la Educación Post-secundaria.

Figura 2. Estructura del sistema educativo de Singapur

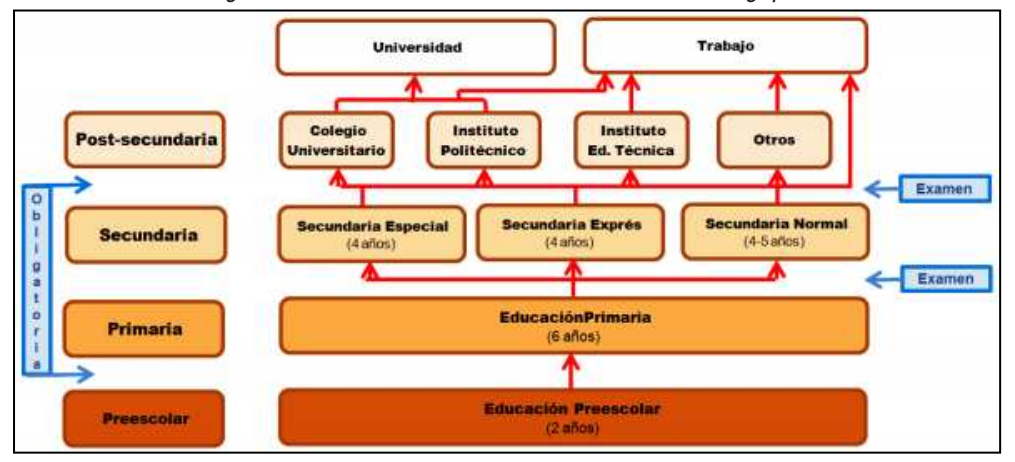

El marco curricular del Método Singapur tiene como centro de aprendizaje la resolución de problemas matemáticos y lo desarrolla a través de cinco componentes básicos: conceptos, habilidades, actitudes, metacognición y procesos (Figura 3).

Figura 3. Marco curricular del Método Singapur (Fuente: Ministerio de Educación de Singapur)

\begin{tabular}{|l} 
O Convicción \\
O Interés \\
O Apreciación \\
o Confianza \\
o Perseverancia
\end{tabular}




\section{EL MÉTODO SINGAPUR PARA EL APRENDIZAJE DE LAS MATEMÁTICAS. ENFOQUE Y CONCRECIÓN DE UN ESTILO DE APRENDIZAJE}

La resolución de problemas se realiza desde situaciones de la vida real y supone un desafío que requiere a los estudiantes hacerse preguntas, contrastar hipótesis y conjeturas y buscar los conceptos matemáticos subyacentes en cada situación. Su objetivo se centra más en la comprensión y en la explicación del proceso que en la obtención del resultado, por lo que se anima a los estudiantes a resolver los problemas de varias maneras y se les proporciona diferentes estrategias y formas de exploración; de esta manera se evita la generación de hábitos rutinarios que asocien de forma mecánica problema y algoritmo.

El Método Singapur se basa en cuatro aspectos metodológicos fundamentales: (1) el enfoque CPA (concreto-pictórico-abstracto), (2) el currículo en espiral, (3) las variaciones sistemática y perceptual y (4) la comprensión relacional frente a la comprensión instrumental.

Con el enfoque CPA los estudiantes construyen sus conocimientos a través de tres niveles de representación graduados por su complejidad: concreto, pictórico y abstracto. En el nivel concreto los estudiantes comienzan a comprender un concepto manipulando materiales y objetos del entorno; en el nivel pictórico avanzan en la comprensión del concepto representándolo mediante dibujos o imágenes; y en el nivel abstracto acaban el proceso de comprensión representándolo mediante signos o símbolos matemáticos. En la figura 4 se observan los tres niveles de representación en un problema de descomposición de un número.

Figura 4. Ejemplo de aplicación enfoque Concreto-Pictórico-Abstracto

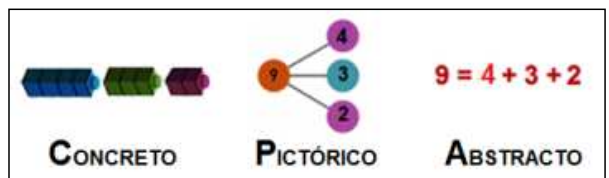

Con el currículo en espiral se trabajan los conceptos a distintos niveles y adaptados a las posibilidades de los estudiantes: se introduce un concepto y se trabaja con él varias veces durante el mismo año y en años posteriores, aumentando gradualmente su complejidad y abstracción. Con el currículo en espiral se refuerzan los conocimientos anteriores sobre los que se sustentan los nuevos conocimientos, manteniendo la jerarquía y las relaciones de los contenidos. En la tabla 1 se observa, a modo de ejemplo, el carácter espiral del tópico de probabilidad en el currículo de Educación Primaria.

Tabla 1. Carácter "espiral" del tópico de la probabilidad en el currículo de Singapur

\begin{tabular}{|c|c|c|c|c|c|c|}
\hline Contenidos & $1^{0}$ & $2^{0}$ & $3^{\circ}$ & $4^{0}$ & $5^{\circ}$ & $6^{0}$ \\
\hline Comparación de dos sucesos: más, menos o igual probable & $\mathrm{x}$ & $\mathrm{x}$ & & & & \\
\hline Clasificación de sucesos: cierto, probable, improbable e imposible & $\mathrm{x}$ & $\mathrm{x}$ & $\mathrm{x}$ & & & \\
\hline Combinaciones & & & $\mathrm{x}$ & $\mathrm{x}$ & $\mathrm{x}$ & $\mathrm{x}$ \\
\hline Comprensión de probabilidad & & & & $\mathrm{x}$ & $\mathrm{x}$ & \\
\hline Cálculo de probabilidades & & & & $\mathrm{x}$ & $\mathrm{x}$ & \\
\hline Hacer predicciones & & & & $\mathrm{x}$ & $\mathrm{x}$ & $\mathrm{x}$ \\
\hline Sucesos compuestos & & & & & & $x$ \\
\hline
\end{tabular}

Con la variación sistemática se presenta a los estudiantes un mismo concepto de diferentes formas con distinto grado de complejidad y abstracción y mediante la variación perceptual los estudiantes interiorizan el concepto de la forma que más les interese. En la figura 5 se muestran tres formas diferentes de descomponer un número en el nivel concreto. 
Figura 5. Ejemplo de variación sistemática

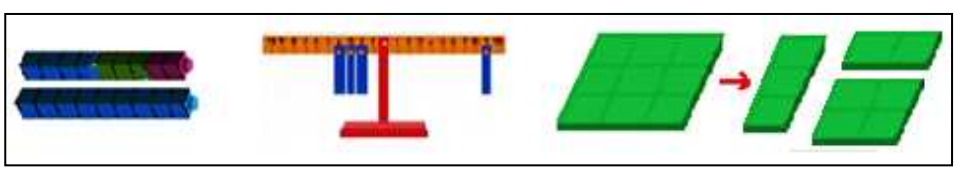

El aprendizaje tradicional, basado en la comprensión instrumental, promueve la memorización de reglas que se aplican a situaciones muy determinadas, mientras que en el Método Singapur, basado en la comprensión relacional, se construyen conceptos para dar respuesta a situaciones de distinta naturaleza que se plantean en la vida diaria.

\section{FUNDAMENTACIÓN TEÓRICA}

El Método Singapur para el aprendizaje de las matemáticas se fundamenta en las ideas de tres importantes investigadores matemáticos de la segunda mitad del siglo XX:

1. Bruner (1915-2016), psicólogo y pedagogo estadounidense representante del cognitivismo en educación, que desarrolló en la década de los 60 la teoría conocida como aprendizaje por descubrimiento. Sus principales aportaciones al Método Singapur son los modos de representación (enactivo, icónico y simbólico) que han derivado en el enfoque CPA y el currículo en espiral.

2. Dienes (1916-2014), matemático húngaro impulsor de la Psicomatemática, que introdujo la utilización de materiales manipulativos, como los bloques lógicos, en el aprendizaje de las matemáticas. Sus principales aportaciones son las variabilidades matemática y perceptual, que en el Método Singapur se concretan en las variaciones sistemática y perceptual.

3. Skemp (1919-1995), matemático y psicólogo británico autor de la "Psicología del aprendizaje de las matemáticas", que investigó la forma en la que los estudiantes construyen los conceptos matemáticos. Su principal aportación al Método Singapur es la comprensión relacional.

Figura 6. Fundamentación teórica del Método Singapur

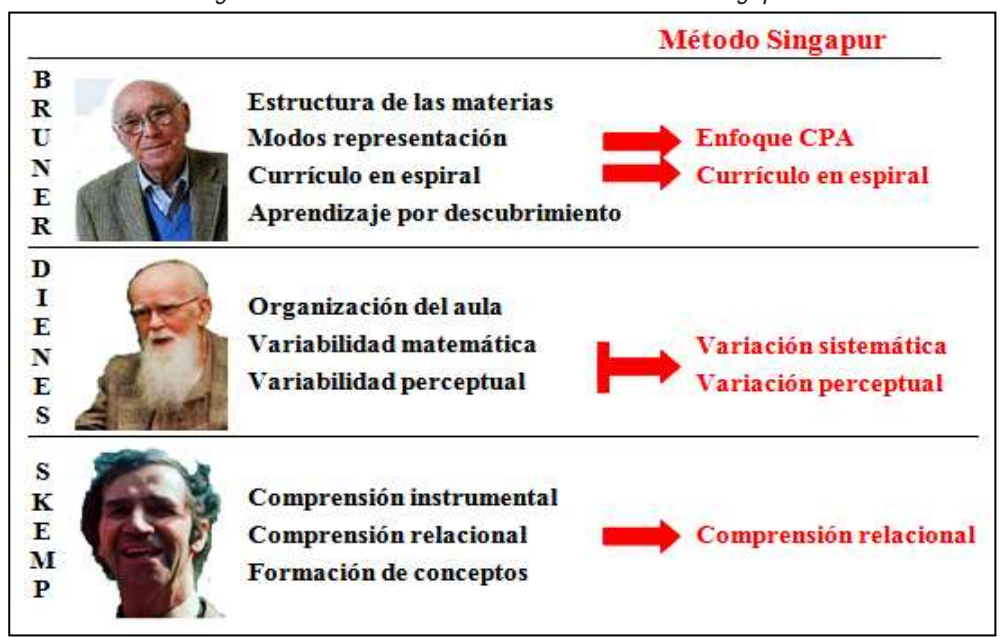

\section{Aportaciones de Jerome Bruner}

Bruner promovió, en la década de los 60, un cambio en el modelo educativo desde el conductismo hacia un enfoque cogniscitivo y simbólico del aprendizaje. Este cambio supuso el paso de los modelos reduccionistas y 


\section{EL MÉTODO SINGAPUR PARA EL APRENDIZAJE DE LAS MATEMÁTICAS. ENFOQUE Y CONCRECIÓN DE UN ESTILO DE APRENDIZAJE}

mecanicistas del aprendizaje memorístico centrados en la figura del docente y que concebían a los estudiantes como receptores pasivos del conocimiento a un aprendizaje entendido como un proceso activo que fomenta la competencia "aprender a aprender" y en el que el estudiante construye su propio proceso de aprendizaje.

Cuatro aspectos del aprendizaje estudiados por Bruner fundamentan teóricamente el Método Singapur: la importancia de la estructura, los modos de representación, el currículo en espiral y la intuición en el aprendizaje por descubrimiento.

a. Estructura de las materias

Con el término estructura, Bruner se refiere a las ideas fundamentales de las materias y a las relaciones que se establecen entre ellas. Considera que el sistema educativo debe proporcionar a los estudiantes una comprensión de la estructura de cada una de las materias porque su comprensión es requisito para la resolución de nuevos problemas.

Señala que un aprendizaje es útil si cumple dos condiciones: se puede aplicar a otras tareas similares y favorece la resolución de tareas futuras. Los psicólogos se refieren a la primera condición como transferencia específica de adiestramiento y a la segunda como transferencia no específica o transferencia de principios y Bruner considera la primera como una ampliación de hábitos, mecanismos o asociaciones y la segunda como el aprendizaje de una tarea general que puede usarse como base para resolver otras tareas.

El estudiante que comprende la estructura de una materia es consciente del conocimiento que tiene sobre su propio funcionamiento cognitivo y de los procedimientos de supervisión y regulación; es decir, es consciente de "Io que sabe" y de "cómo lo sabe", los dos aspectos de la metacognición, como producto y como proceso.

La estructura de Bruner se refleja de forma general en el marco curricular del Método Singapur, que tiene como centro de aprendizaje de las matemáticas la resolución de problemas, y de forma específica en uno de sus componentes, la metacognición, que comprende dos procesos: monitorización de los pensamientos y autorregulación del aprendizaje.

\section{b. Modos de representación}

Bruner sostiene que los estudiantes en su desarrollo cognitivo utilizan técnicas o destrezas para representar los estímulos del entorno a partir de la acción, de las imágenes mentales y de los símbolos; esta idea da lugar a tres modos de representación en función de su grado de desarrollo cognitivo: enactivo, icónico y simbólico.

En el modo enactivo los estudiantes representan las situaciones mediante una respuesta motriz adecuada, en el modo icónico representan con imágenes los objetos sin actuar directamente sobre ellos y en el modo simbólico expresan sus experiencias de forma abstracta mediante símbolos arbitrarios que representan objetos, situaciones o ideas.

Los tres modos de representación se relacionan entre sí evolutivamente: se desarrollan en ese orden y cada uno de ellos depende del anterior. Y aunque los tres modos pueden actuar de forma simultánea, la práctica de cada uno de ellos es necesaria para llevar a cabo la transición al siguiente, por lo que la mejor forma de presentar los contenidos es en una secuencia que comience por una representación enactiva, continúe por una representación icónica y termine en una representación simbólica.

El Método Singapur concretó esta progresión del desarrollo cognitivo de Bruner en los tres niveles del enfoque CPA: en el nivel concreto los estudiantes, a través del material concreto, indagan, descubren y aplican conceptos matemáticos que facilitan la resolución de problemas; en el nivel pictórico, dibujan e interpretan la información a partir de modelos gráficos representando los datos y las relaciones que ayudan a visualizar y resolver los problemas; y en el nivel abstracto, desarrollan los problemas presentados utilizando signos y símbolos matemáticos que traducen la experiencia concreta y pictórica

Este enfoque pretende que en un principio los estudiantes experimenten con material concreto para deducir ciertos conceptos, que a partir de estos nuevos conceptos realicen generalizaciones para caracterizar la información mediante representaciones pictóricas y, por último, que se apoyen en las interrelaciones entre los niveles de desarrollo anteriores para llegar al nivel de la abstracción y realizar representaciones simbólicas matemáticas que les permitan una mayor capacidad para analizar y solucionar nuevas situaciones. 
c. Currículo en espiral

Bruner se apoya en este carácter progresivo del desarrollo cognitivo para evidenciar el carácter espiral de los currículos y desarrollar los contenidos de forma progresiva y reiterativa, aumentando su complejidad y adaptándolos a la edad de los estudiantes. Justifica el currículo en espiral afirmando que los estudiantes pueden aprender cualquier contenido siempre que se adapte a sus capacidades, "se respeten sus maneras de pensar [...] y si se le reta lo suficiente [...] es posible introducir al niño en edad temprana a las ideas y estilos que harán de él un hombre educado" (Bruner, 1960, p. 80).

El currículo en espiral se caracteriza por una revisión continua de los conceptos, de forma que los estudiantes tratarán los conceptos generales en varios momentos diferentes, pero cada vez en forma más abstracta y compleja. Sin embargo, a menudo el desarrollo en espiral del currículo falla en su implementación (Gibbs, 2014) reduciéndolo a una simple repetición de conceptos; para evitarlo se requiere del maestro flexibilidad y reflexión sobre los diferentes ritmos de aprendizaje de los estudiantes que siguen procesos autónomos, independientes y variables.

El Método Singapur adoptó el currículo en espiral presentando los contenidos matemáticos de forma gradual: en los primeros años se incorporan actividades lúdicas y se posponen las definiciones y conceptos para años posteriores; se permite de esta forma que los estudiantes se apropien de los conceptos a medida que estén preparados para adoptarlos e interiorizarlos.

\section{d. La intuición en el aprendizaje por descubrimiento}

Bruner sostiene que los estudiantes llegan de forma intuitiva a formulaciones provisionales y que posteriormente, a través de lo que van aprendiendo, validan o rechazan esas formulaciones y afirma que el pensamiento intuitivo, entendido como la habilidad para conocer, comprender o percibir algo sin la intervención de la razón, "es un rango esencial y muy descuidado del pensamiento productivo, no sólo en las disciplinas académicas formales, sino también en la vida cotidiana” (Bruner, 1960, pág. 21).

Para adquirir nuevos conocimientos, los estudiantes deben plantear hipótesis y conjeturas provisionales sin miedo a equivocarse, porque su aprendizaje será más significativo si parte de sus propias investigaciones y exploraciones y no solo de los conocimientos que le proporcionan el maestro y los libros de texto.

El pensamiento intuitivo, a diferencia del pensamiento analítico, no se rige por estructuras precisas y exactas, pero es importante en el aprendizaje de las matemáticas porque "hacer matemáticas implica descubrir, y la conjetura es el principal camino para el descubrimiento" (NCTM, 2003, p.60) y "vislumbrar más allá de lo que se percibe, ver alguna regularidad y plantear conjeturas es el corazón de la inducción" (Cañadas, 2007).

Desde esta perspectiva la intuición va unida a la inducción y Bruner (2001) considera que el aprendizaje en el aula puede tener lugar inductivamente, es decir, pasando a través de la generalización de lo particular a la formulación de un principio general, de lo concreto a lo abstracto.

La intuición se concretó en el Método Singapur al proporcionar a los estudiantes situaciones y problemas que les estimulan a plantear conjeturas para descubrir por sí mismos los conceptos, relaciones y procedimientos, como partes de un todo organizado.

\section{Aportaciones de Zoltan Dienes}

Dienes anima a los maestros a pasar de una enseñanza de las matemáticas basada en cálculos matemáticos a una enseñanza basada en la comprensión: "se hace necesario educar a los niños en la comprensión de la matemática y de sus aplicaciones. Esto se convierte en una parte esencial de nuestra cultura" (Dienes, 1969, p.5). De esta forma, la enseñanza de las matemáticas no debe limitarse a simples algoritmos ajenos a la vida diaria, sino que debe fomentar en los estudiantes un interés por las matemáticas que les permita dar respuesta a sus propias necesidades y a las situaciones con las que se enfrentan, a partir del juego y del uso de la lógica.

Las aportaciones de Dienes al Método Singapur se centran en la organización del aula y en las variabilidades matemática y perceptual. 


\section{EL MÉTODO SINGAPUR PARA EL APRENDIZAJE DE LAS MATEMÁTICAS. ENFOQUE Y CONCRECIÓN DE UN ESTILO DE APRENDIZAJE}

\section{a. Organización del aula}

El modelo de enseñanza no se cambia con constantes reformas de los currículos y planes de estudio, es preciso cambiar las estrategias, los procedimientos y la organización de las aulas; esto supone un importante desafío para los maestros que "esperamos se esforzarán en pasar de una situación de enseñanza a una situación de aprendizaje" (Dienes, 1969, p.7).

Este cambio implica para el maestro nuevas formas de presentar las situaciones mediante tareas motivadoras adaptadas al desarrollo de los estudiantes, y para los estudiantes implica nuevas formas de resolver las tareas para contextualizarlas e interiorizarlas. En otras palabras, se ha de reorganizar el aula para pasar de enseñar matemáticas a los estudiantes a que los estudiantes aprendan matemáticas orientados por el maestro 0 de forma espontánea.

En esta nueva organización el maestro debe considerar cada situación para establecer la forma de trabajo: individual, en pequeños grupos o de toda la clase; Dienes propone introducir los conceptos nuevos durante un período corto de tiempo con toda la clase y que después los estudiantes exploren el concepto de forma individual 0 en pequeño grupo. Además, el maestro debe respetar los procesos de aprendizaje de los estudiantes sin coartar sus iniciativas con premios o castigos; sin la imposición de premios y castigos, los estudiantes muestran un mayor interés por la investigación y el descubrimiento del entorno y aprenden matemáticas por sí mismos.

Otro aspecto importante en la organización del aula es la discusión entre estudiantes; cuando un estudiante comete un error, es preferible que se lo advierta un compañero y no el maestro, porque entre ellos pueden discutir la situación en un plano de igualdad, justificando y argumentando sus posturas y "es infinitamente mejor inclinar a los niños a que busquen la verdad, antes de que lo haga la autoridad de la persona encargada de administrarla, el maestro, por ejemplo" (Dienes, 1969, p.9). Los debates entre iguales proporcionan un interesante modelo de aprendizaje social en el aula, por lo que debe fomentarse la participación y la comunicación a través de exposiciones verbales donde el maestro modere y guíe la discusión matemática hacia el rigor a partir de los argumentos de los estudiantes (Mariotti, 2009).

Dienes considera también que para que el aprendizaje sea realmente activo, el aula debe disponer de suficiente material que debe estar ordenado y al alcance de los estudiantes, por lo que el maestro debe planificar adecuadamente las actividades y el reparto del material.

Esta organización del aula, en la que los estudiantes aprenden de forma activa y cooperativamente, supone un nuevo reto y desafío para el maestro que debe adaptarse a la nueva situación y renunciar a su papel de autoridad y actuar como moderador y orientador, ejerciendo la responsabilidad del aprendizaje de sus alumnos desde el acompañamiento y no desde la autoridad. La tarea del maestro es "hacer sugerencias en el momento oportuno, pero no dándole el carácter de orden, ya que el estudiante pensará que está realizando la tarea resuelta por el docente y no será desafiante para él encontrar una solución" (Calderón, 2014, p.37). En este sentido Dienes advierte al maestro que su forma de pensar y actuar como adulto muchas veces no coincide con la de sus alumnos, que no existe una forma única de resolver un problema y que a menudo los estudiantes proponen soluciones distintas a las que él hubiera elegido.

El Método Singapur concreta estas ideas al considerar que contextos adecuados con los que "enseñar a aprender" y recursos y materiales didácticos apropiados mejoran la enseñanza y ayudan a la construcción de situaciones de aprendizaje.

\section{b.- Variabilidad matemática y variabilidad perceptual}

En este contexto, Dienes (1978) aporta dos términos muy significativos en el aprendizaje de las matemáticas: la variabilidad matemática y la variabilidad perceptual. Con la variabilidad matemática establece que un concepto ha de ser presentado de diversas formas variando tanto como sea posible su estructura para que se distingan todas las características matemáticas implicadas y con la variabilidad perceptual afirma que ha de variarse suficientemente el marco experimental a partir del cual se desarrollan ideas y procesos para propiciar la generalización y la abstracción que permitan al estudiante construir su propio conocimiento. 
El Método Singapur ha adoptado estos conceptos denominándolos variación sistemática y variación perceptual. La variación sistemática plantea presentar los conceptos de distintas maneras y con distinto grado de profundidad y la variación perceptual permite a los estudiantes interiorizar un concepto de la forma que más les interese.

El método Singapur propone generar pequeñas variaciones en la forma de presentar un determinado problema para evitar la repetición y promueve que el estudiante descubra distintas estrategias y procedimientos para resolver el problema y elija de forma autónoma la que más se acerque a sus intereses. Pretende que los estudiantes resuelvan tareas y problemas matemáticos de forma sistemática y reiterada, pero con pequeños ajustes graduales en su dificultad y en su resolución; un número suficiente de ejemplos, pero no excesivo, permitirá a los estudiantes a construir sus propias estrategias y no se limitarán a aprender de forma mecánica y memorística las respuestas.

De esta manera, el Método Singapur no enseña procedimientos para resolver problemas como en la enseñanza tradicional, sino que promueve que los estudiantes tomen las mejores decisiones en función de las circunstancias. Para ello los anima a visualizar el problema y generar estrategias mentales distintas, fomentando así la flexibilidad necesaria para aplicar la mejor estrategia en cada situación.

\section{Aportaciones de Richard Skemp}

Skemp estudió los aspectos psicológicos del aprendizaje de las matemáticas centrando sus ideas en la comprensión y sus tipos y en la formación de conceptos.

a. Comprensión instrumental y comprensión relacional

Históricamente la comprensión se asociaba con el conocimiento hasta que Skemp (1976) diferenció ambos términos señalando dos categorías de la comprensión matemática: la comprensión instrumental y la comprensión relacional. Definió la primera como "saber hacer" y la segunda como."saber por qué hacerlo".

La comprensión instrumental implica conocer una serie de planes preestablecidos para desarrollar paso a paso tareas matemáticas; estos planes se aprenden fácilmente y proporcionan al estudiante una respuesta rápida para determinadas tareas. Por su parte, la comprensión relacional se caracteriza por el dominio de unas estructuras conceptuales que permiten construir diferentes planes para desarrollar tareas matemáticas; estas estructuras son más difíciles de aprender pero son más adaptables a tareas nuevas, por lo que son más aconsejables en la educación matemática.

El aprendizaje basado en la comprensión instrumental promueve la memorización de una serie de reglas para aplicar a determinadas situaciones para llegar a la respuesta correcta y se aprende para una determinada situación y para un determinado momento; sin embargo, el aprendizaje basado en la comprensión relacional se refiere a la construcción de conceptos para dar respuesta a diferentes situaciones y problemas que se plantean en la vida diaria y se aprende para toda la vida ya que puede ser adaptado a otras situaciones y momentos.

El Método Singapur, siguiendo las orientaciones de Skemp, fomenta la comprensión relacional en el aprendizaje de las matemáticas frente a la comprensión instrumental de la enseñanza tradicional. Pero, para elaborar los planes y estrategias que requiere la comprensión relacional y que le permiten resolver problemas matemáticoss, el estudiante debe construir los conceptos que subyacen en el problema.

\section{b. Formación de conceptos}

Para Skemp las matemáticas son un esquema de conceptos que se organizan en niveles de abstracción, de forma que un estudiante solo puede asimilar conceptos de orden más elevado por medio de ejemplos.

Los estudiantes adquieren continuamente conocimientos directamente del entorno, pero las matemáticas " $n o$ pueden aprenderse directamente del entorno cotidiano, sino sólo de manera indirecta desde otros matemáticos" (Skemp, 1980, pág. 36); los estudiantes deben apoyarse en procesos matemáticos previos provenientes de la abstracción y generalización de generaciones anteriores. Esto supone una gran ventaja ya que en poco tiempo pueden aprenderse conceptos que han tardado siglos en desarrollarse. 


\section{EL MÉTODO SINGAPUR PARA EL APRENDIZAJE DE LAS MATEMÁTICAS. ENFOQUE Y CONCRECIÓN DE UN ESTILO DE APRENDIZAJE}

Además, la construcción de conceptos requiere dos funciones: integrar conocimientos ya existentes y transformar dichos conocimientos en un instrumento mental. Estas funciones, se concretan en dos principios en el aprendizaje de las matemáticas que, aunque objetivos y aparentemente simples, requieren una gran reflexión por parte del maestro:

Para presentar a un estudiante conceptos de un grado superior a los que ya tiene, no basta con definirlos, es preciso prepararle mediante una serie adecuada de ejemplos.

Los ejemplos son también conceptos, por lo que es preciso asegurarse de que ya están formados en el estudiante.

Con frecuencia, los maestros y los libros de texto incumplen el primer principio al introducir los conceptos nuevos, no con ejemplos, sino mediante definiciones que, aunque sean sencillas, están hechas para el maestro, resultan complicadas para el estudiante y muchas veces le provocan frustración. Y para no incumplir el segundo principio, el maestro, al proponer un ejemplo nuevo, debe asegurarse de los conceptos previos que posee el estudiante.

Así pues, un reto para el maestro es elegir bien los ejemplos para que, a partir de ellos, el estudiante pueda construir los nuevos conceptos. Sin embargo, la elección adecuada de ejemplos es más difícil de lo que parece porque para poder generalizar y abstraer a partir de ellas el concepto, todos los ejemplos deben tener en común las propiedades del concepto, y no otras.

La mayoría de los conceptos matemáticos, en sus acepciones más simples, se adquieren a temprana edad, pero el maestro debe estar atento para reafirmarlos o corregirlos mediante más ejemplos y actividades, porque la interiorización de errores en un concepto dificultará después el aprendizaje de conceptos más abstractos.

Desde esta perspectiva, en la construcción de conceptos existe una dependencia: para interiorizar un determinado concepto, el estudiante debe recurrir a conceptos interiorizados previamente (Calderón, 2014). Esta dependencia es mayor en matemáticas que en otras disciplinas, porque en matemáticas los conceptos están interconectados mientras que en otras disciplinas la mayoría de los conceptos se encuentran en bloques estancos. "Uno comprende la geografía de África incluso si ha omitido la de Europa [...], pero entender álgebra sin antes haber comprendido aritmética, realmente es imposible, pues gran parte del álgebra que aprendemos en la escuela es aritmética generalizada" (Skemp, 1980, p.38).

El Método Singapur, frente a la comprensión instrumental de la enseñanza tradicional, fomenta la comprensión relacional en el aprendizaje de las matemáticas. En la comprensión relacional el estudiante, para elaborar los planes y estrategias que le permiten resolver los problemas matemáticos que se le plantean, debe construir las estructuras conceptuales que subyacen en el problema. Para ello, siguiendo las teorías sobre la formación de conceptos de Skemp, propone la utilización de ejemplos graduales que se han de sustentar en conceptos anteriores del estudiante.

\section{CONCLUSIONES}

El Método Singapur ha sabido adaptar unas teorías de la segunda mitad del siglo pasado a las características propias de sus estudiantes y a la idiosincrasia del país para hacer de su sistema educativo uno de los de mejor rendimiento a nivel mundial.

La solución de países occidentales, como España y Portugal, no es copiar paso a paso esta metodología, sino adaptarla también a nuestras particularidades buscando en nuestros estudiantes, más que la obtención de buenos resultados académicos, la adquisición de competencias que les permitan enfrentarse con garantías a las diferentes situaciones de la vida diaria.

Aspectos como entender la educación como una inversión y no un gasto, mejorar la formación y el perfeccionamiento de nuestros maestros, consensuar un currículo que prime la calidad sobre la cantidad y que fomente la comprensión y la creatividad frente a la memorización y la repetición o ceder más autonomía a los centros y procurarles más recursos son aspectos a copiar por nuestras administraciones educativas. 
Renunciar a su papel de autoridad y protagonista para adoptar el de moderador y orientador, cambiar la metodología basada en la aplicación de algoritmos a otra basada en la comprensión, favorecer la creatividad y la intuición presentando situaciones del propio contexto, estimular a los estudiantes a construir nuevos conocimientos y su propio aprendizaje, fomentar el trabajo colaborativo y la discusión entre iguales, utilizar materiales y recursos didácticos, en definitiva, "enseñar menos y aprender más" y fomentar la competencia "aprender a prender" son desafíos y retos a los que debe enfrentarse el maestro en este proceso de adaptación al Método Singapur.

Sin embargo, otros componentes del sistema educativo de Singapur, como la alta competitividad del sistema y la canalización temprana de los estudiantes, deberían ser estudiados de forma detenida y profunda por nuestros sistemas educativos en su proceso de adaptación.

\section{REFERENCIAS BIBLIOGRÁFICAS}

Alves, M. (2018). Learning styles in mathematics- A quantitative research on 10th grade portuguese students. Revista Estilos de Aprendizaje, 11(22), 83-108.

Bruner, J.S. (1960). The Process of Education. Harvard University Press, Cambridge, MA.

Bruner, J.S. (2001). El proceso mental en el aprendizaje. Madrid: Ediciones Narcea

Calderón, P. (2014). Precepciones de los y las docentes del primer ciclo básico, sobre la implementación del método Singapur en el Colegio Mario Bertero Cevasco de la Comuna de Isla de Maipo. Tesis Doctoral: Universidad de Chile

Cañadas, M.C. (2007). Descripción y caracterización del razonamiento inductivo utilizado por estudiantes de educación secundaria al resolver tareas relacionadas con sucesiones lineales y cuadráticas. Tesis Doctoral: Universidad de Granada. Recuperado de: http://digibug.ugr.es/bitstream/10481/1581/1/16737556.pdf

Dienes, Z.P. (1969). Building Up Mathematics. London: Hutchison Education.

Dienes, Z.P. (1978). La matemática moderna en la enseñanza primaria. Barcelona: Teide.

Gibbs, B.C. (2014). Reconfiguring Bruner: Compressing the Spiral Curriculum. Phi Delta Kappan, 95(7), 41-44. INEE (2016). Resultados del Estudio Internacional de Tendencias en Matemáticas y Ciencias TIMSS 2015. Madrid: Ministerio de Educación, Ciencia y Deporte.

Mariotti, M.A. (2009). Artifacts and signs after a Vygotskian perspective: the role of the teacher". ZDM: The international journal on Mathematics Education, 41(4), 427-440.

Mosquera, D.R. y Salazar, N.J. (2014). Estilos de aprendizaje: "Pensamientos e inquietudes de los estudiantes sobre el aprendizaje de las matemáticas". Revista Estilos de Aprendizaje, 7(13), 2-25.

National Council of Teachers of Mathematics (2003). Principios y estándares para la educación matemática. Sevilla: SAEM Thales

OCDE (2016). Pisa 2015: Resultados clave. Paris: OCDE

Santaolalla, E. (2009). Matemáticas y estilos de aprendizaje. Revista Estilos de Aprendizaje, 4(2), 56-69.

Skemp, R. R. (1976). Relational understanding and instrumental understanding. Mathematics teaching, 77(1), $20-$ 26.

Skemp, R. R. (1980). Psicología del aprendizaje de las matemáticas. Madrid: Morata. 
